\title{
Questes
}

Revue pluridisciplinaire d'études médiévales

Journée d'étude 1 - Trier, classer, organiser | 2022

Trier, classer, organiser

\section{Les Quinze Signes du Jugement dernier selon Pierre le Mangeur : étude des différentes versions françaises}

\section{Louis-Patrick Bergot}

\section{OpenEdition}

Journals

Édition électronique

URL : https://journals.openedition.org/questes/6067

DOI : 10.4000/questes.6067

ISSN : 2109-9472

Éditeur

Les Amis de Questes

Édition imprimée

Pagination : 229-244

ISSN : 2102-7188

Référence électronique

Louis-Patrick Bergot, « Les Quinze Signes du Jugement dernier selon Pierre le Mangeur : étude des différentes versions françaises », Questes [En ligne], Journée d'étude 1 - Trier, classer, organiser | 2022 , mis en ligne le 31 janvier 2022, consulté le 21 février 2022. URL : http://journals.openedition.org/ questes/6067; DOI : https://doi.org/10.4000/questes.6067 


\title{
Les Quinze Signes du Jugement dernier selon Pierre le Mangeur : étude des différentes versions françaises
}

\author{
Louis-Patrick BERGOT
}

Sorbonne Université

La légende des Quinze Signes fait l'inventaire des quinze signes qui précéderont le Jugement dernier durant une période de quinze jours. Les racines de cette légende sont complexes et remontent, d'une part à la littérature apocalyptique judéo-chrétienne, d'autre part au folklore irlandais ${ }^{1}$. Au cours du Moyen Âge, elle s'est développée et a connu un succès croissant. Comme l'a fait remarquer Hugh Shields, la légende des quinze signes du Jugement dernier était « aussi populaire au Moyen Âge qu'elle est méconnue de nos jours ${ }^{2} »$. Force est de constater que les médiévaux y accordaient plus de crédit que nous ne saurions l'imaginer. Même saint Thomas d'Aquin en parle dans sa Somme théologique, au moment d'évoquer les fins dernières de l'humanité ${ }^{3}$. Aux xiv et $\mathrm{xv}^{\mathrm{e}}$ siècles, plusieurs grands textes de la littérature française $\mathrm{y}$ font allusion, par exemple le Roman de Fauvel: «[...] se l'Escripture ne ment, / Les signes perent que pres estre / Devons du temps en quoi doit

\footnotetext{
${ }^{1}$ Sur les origines de cette légende, voir William Heist, The Fifteen Signs before Doomsday, East Lansing (Mi.), Michigan State College Press, 1952.

2 « [It] was as popular in the Middle Ages as it is unfamiliar today »: Hugh Shields, «Les Quinze Signes descendus en Angleterre. A medieval legend in decline », French Studies, 18, 1964, p. 112-122, cit. p. 112.

${ }^{3}$ Saint Thomas d'Aquin reprend la version de Pierre Damien dans la question $73 \mathrm{du}$ Supplément: voir saint Thomas d'Aquin, Somme théologique. Suppl., questions 6974, L'au-delà, éd. et trad. Jean-Dominique Folghera, Paris/Tournai, Desclée/Éditions de la Revue des Jeunes, 1935.
} 
nestre/ L'anemy de Crestienté ${ }^{4} »$. François Villon mentionne aussi la légende dans son Lais: le poète laissera «aux Mendïans / Aux Filles Dieu et aux Beguines », outre de «Savoureux morceaulx et fryans, / Chappons, flaons, grasses gelines», le privilège de «prescher les .xv. signes ${ }^{5} \gg$. Dans les années 1530, la légende donnera naissance à une parodie d'inspiration rabelaisienne ${ }^{6}$. Peu de temps après, Joachim du Bellay l'évoquera encore dans sa célèbre Deffence et illustration de la langue françoyse ${ }^{7}$.

Étant donné l'immense succès de cette légende, on ne sera guère surpris de découvrir qu'il existait au Moyen Âge un nombre considérable de versions latines. La critique a distingué plusieurs modèles latins, chacun ayant sa propre liste de signes :

\section{Tableau 1}

Les différents modèles latins de la légende des quinze signes

\begin{tabular}{|l|l|}
\hline \multicolumn{1}{|c|}{ Modèle } & \multicolumn{1}{c|}{ Date approximative } \\
\hline Pseudo-Bède & avant le $\mathrm{X}^{\mathrm{e}}$ siècle \\
\hline Pierre Damien & milieu du XI ${ }^{\mathrm{e}}$ siècle \\
\hline Pierre le Mangeur & milieu du XII ${ }^{\mathrm{e}}$ siècle \\
\hline Version $\mathrm{x}$ & avant la fin du XII ${ }^{\mathrm{e}}$ siècle \\
\hline Version composite/Legenda aurea & $1261-1266$ \\
\hline
\end{tabular}

\footnotetext{
${ }^{4}$ Le Roman de Fauvel, éd. Armand Strubel, Paris, LGF, coll. «Lettres gothiques », 2012, v. 3989-3992.

${ }^{5}$ François Villon, Le Lais Villon, éd. Jean Rychner et Albert Henry, Genève, Droz, 1977, t. 1, v. 249-253.

${ }^{6}$ Pour une édition de ce texte, voir Hugh Shields, «Les Quinze Signes... », art. cit.

${ }^{7}$ Voir Joachim du Bellay, La Deffence, et illustration de la Langue Françoyse, Paris, Arnoul l'Angelier, 1549, p. 36.
} 
Dans le cadre de ce travail, nous voudrions nous arrêter sur la version de Pierre le Mangeur, qui fut l'une des plus répandues, et sur l'ensemble des traductions françaises qui en sont issues. L'étude de ces traductions démontre la malléabilité de la légende et illustre les métamorphoses que toute liste est vouée à connaître au Moyen Âge en vertu de la transmission manuscrite.

\section{La légende selon Pierre le Mangeur}

Le texte latin sur lequel porte notre étude se trouve dans l'Historia scholastica de Pierre le Mangeur ${ }^{8}$ (1169-1173). Donnons d'abord une brève description des Quinze Signes selon cet auteur ${ }^{9}$ :

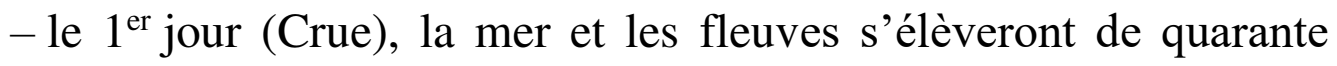
coudées à la manière d'un mur ;

- le $2^{\mathrm{e}}$ jour (Décrue), ils redescendront à leur plus bas niveau ;

- le $3^{\mathrm{e}}$ jour (Bêtes marines), les poissons et les bêtes marines pousseront un cri que seul Dieu pourra comprendre ;

- le $4^{\mathrm{e}}$ jour (Incendie aquatique), les cours d'eau s'embraseront ;

- le $5^{\mathrm{e}}$ jour (Rosée sanglante), les herbes et les arbres produiront une rosée sanglante ;

- le $6^{\mathrm{e}}$ jour (Chute des édifices), tous les édifices seront détruits ;

- le $7^{\mathrm{e}}$ jour (Lutte des pierres), les pierres se scinderont en trois parties, avant de se frapper mutuellement ;

\footnotetext{
${ }^{8}$ Pierre le Mangeur, Historia Scholastica, «Historia Evangelica», CXLI. Pour le texte, voir PL, t. CXCVIII, col. 1611. Sur l'Historia scholastica, on pourra lire l'ouvrage récent de Mark J. Clark, The Making of the Historia scholastica, 1150-1200, Toronto, Pontifical Institute of Mediaeval Studies, 2015.

${ }^{9}$ Entre parenthèses, nous résumons chacun des signes à l'aide de mots clés (Crue, Décrue, etc.), afin de pouvoir par la suite désigner les signes indépendamment de leur ordre chronologique, qui souvent varie.
} 
- le $8^{\mathrm{e}}$ jour (Séisme), il y aura un tremblement de terre, tel qu'on n'en a jamais connu ;

- le $9^{\mathrm{e}}$ jour (Nivellement), les collines et les vallées seront nivelées ;

- le $10^{\mathrm{e}}$ jour (Hommes fous), les hommes sortiront des cavernes et se mettront à courir comme s'ils étaient fous, sans parvenir à communiquer ;

- le $11^{\mathrm{e}}$ jour (Ossements), les ossements des morts surgiront hors des tombeaux ;

- le $12^{\mathrm{e}}$ jour (Chute des étoiles), les étoiles tomberont du ciel ;

- le $13^{\mathrm{e}}$ jour (Mort universelle), tous les hommes trouveront la mort ;

- le $14^{\mathrm{e}}$ jour (Conflagration), la terre et la mer s'embraseront ;

- le $15^{\mathrm{e}}$ jour (Résurrection), un nouveau ciel et une nouvelle terre seront créés, et tous les hommes ressusciteront pour assister à la Parousie et au Jugement dernier.

La liste de Pierre le Mangeur reprend celle du Pseudo-Bède, que les médiévaux attribuaient par erreur à Bède le Vénérable ${ }^{10}$. Du PseudoBède à Pierre le Mangeur, les changements sont peu nombreux. Le $3^{e}$ signe du Pseudo-Bède fut supprimé ${ }^{11}$, avançant d'un jour les signes suivants (4 à 15$)$. Un $15^{\mathrm{e}}$ signe (Résurrection) fut ajouté. Enfin, les $12^{\mathrm{e}}$ et

\footnotetext{
${ }^{10}$ La version du Pseudo-Bède apparaît au sein des Collectanea Pseudo-Bedae, un ensemble de textes apocryphes qu'une édition protestante de 1563 attribuait encore à Bède le Vénérable, l'auteur de l'Historia ecclesiastica gentis Anglorum (c. 731). Les Quindecim signa du Pseudo-Bède circulaient par ailleurs dans des copies indépendantes dès le $\mathrm{X}^{\mathrm{e}}$ siècle (date des plus anciens témoins). Pour le texte des Quinze Signes, voir Collectanea Pseudo-Bedae, éd. Matha Bayless et Michael Lapidge, Dublin, School of Celtic Studies, 1998, § 356-371, p. 178. Le texte figurait déjà dans la Patrologie latine : PL, t. XCIV, col. 555.

${ }^{11}$ Le $3^{\mathrm{e}}$ signe annonce que le niveau de l'eau, après être monté, puis descendu, reviendra à la normale. Ce $3^{\mathrm{e}}$ signe n'était pas pourvu de ce merveilleux qui caractérise les autres signes : c'est donc naturellement que Pierre le Mangeur l'a rejeté.
} 
$13^{\mathrm{e}}$ signes du Pseudo-Bède (Chute des étoiles et Ossements) furent inversés : avec le décalage, ils devinrent respectivement les $12 \mathrm{e}$ et $11^{\mathrm{e}}$ signes.

S'il est probable que l'inversion des signes 12 et 13 soit due à la transmission manuscrite, la suppression du signe 3 et l'ajout du signe 15, en revanche, répondent à des préoccupations eschatologiques. Le $15^{\mathrm{e}}$ signe du Pseudo-Bède ayant désormais lieu le $14^{\mathrm{e}}$ jour, cela permettait de ne pas terminer la liste sur l'embrasement de l'univers.

Notons enfin que la version de Pierre le Mangeur est à l'origine d'un autre modèle, repris ensuite par Jacques de Voragine pour sa célèbre Légende dorée. Ce nouveau modèle est le résultat d'une synthèse entre celui de Pierre le Mangeur, qui sert pour ainsi dire de «socle», et celui de Pierre Damien ${ }^{12}$, duquel plusieurs passages sont extraits en guise de « compléments ». La version de Pierre le Mangeur est donc au carrefour des diverses traditions latines.

À notre connaissance, il existe au moins cinq traductions françaises de cette version. Trois d'entre elles ne se trouvent que dans un ou deux manuscrits, alors que les deux autres appartiennent à des ouvrages de plus grande ampleur, le Miroir historial et le Dialogue du père et du fils, qui l'un comme l'autre furent transmis par un plus grand nombre de témoins. Nous passerons en revue ces cinq traductions, dans l'ordre suivant :

\footnotetext{
${ }^{12}$ La version attribuée au cardinal Pierre Damien (c. 1007-1072) se trouve dans le quatrième et dernier chapitre d'un court traité eschatologique, intitulé De Novissimis et Antichristo : PL, t. CXLV, col. 840-842.
} 


\section{Tableau 2}

Les cinq traductions françaises de la version de Pierre le Mangeur

\begin{tabular}{|l|l|l|l|}
\hline \multicolumn{1}{|c|}{ Version } & Mss. & \multicolumn{1}{|c|}{ Date } & \multicolumn{1}{c|}{ Édition } \\
\hline Miroir historial de Jean de Vignay & 8 & c. 1332 & (inédit) \\
\hline Londres, BL, Add. 47682, fol. 40v-42r & 1 & $c .1327-1335$ & Pickering (1971) \\
\hline Montpellier, BIU, H 043, fol. 51vb-52a & 1 & milieu du XIV & (inédit) \\
\hline « Selon ce que nostre Sire... » & 2 & fin du XIII & Mantou (1967) \\
\hline Dialogue du père et du fils & $\approx 25$ & milieu du XIII & (inédit) \\
\hline
\end{tabular}

\section{Des traductions plus ou moins fidèles}

\section{Le Miroir historial de Jean de Vignay}

Dans les années 1330, Jean de Vignay traduisit le Speculum historiale de Vincent de Beauvais, qui avait repris la version de Pierre le Mangeur pour l'inclure dans le tome 32 de son encyclopédie ${ }^{13}$. Parmi la trentaine de manuscrits qui préservent le Miroir historial, seuls huit contiennent la légende des Quinze Signes ${ }^{14}$. La traduction qu'en donne Jean de Vignay est très littérale : dans le signe 3 par exemple, le terme belluae («les bêtes ») est traduit par «belues », là où d'autres traducteurs préfèrent dire de quel monstre il s'agit, comme une baleine, une sirène, etc.

\footnotetext{
${ }^{13}$ Vincent de Beauvais, Speculum historiale, XXXII, 110 («De adventu iudicis ac signis preambulis »). Pour le texte, consulter http://atilf.atilf.fr/bichard.

${ }^{14}$ Sur le Miroir historial, dont Mattia Cavagna prépare l'édition, on consultera : Laurent Brun et Mattia Cavagna, "Pour une édition du Miroir historial de Jean de Vignay », Romania, 124, 2006, p. 378-428. Le texte sur les Quinze Signes se trouve dans les huit manuscrits suivants (nous reprenons les sigles proposés ibid.) : N3, T2, GH, EP4, C4, G4, Or4 et Tc6.
} 
La Bible de Holkham (Londres, British Library, Add. 47682, fol. 40v-42r)

À la même époque (c. 1327-1335), un livre à peintures fut confectionné en Angleterre. Ce manuscrit, qui fait partie des plus beaux modèles du genre, provient de la bibliothèque du Holkham Hall et se trouve désormais à la British Library, sous la cote Add. 47682. Avec ses 42 feuillets, la «Bible de Holkham» est structurée en forme de triptyque: le manuscrit s'ouvre sur plusieurs épisodes de la Genèse (fol. 2r-9r), se poursuit par une succession de scènes évangéliques (fol. 10r-38r) et s'achève sur une description des quinze signes du Jugement dernier (fol. 39r-42v). Du début à la fin du manuscrit, la mise en page met en valeur un ensemble de miniatures sans cadre, surmontées de légendes anglo-normandes. C'est aux fol. 40v-42r que se trouve la traduction anglo-normande de Pierre le Mangeur ${ }^{15}$. Elle offre par ailleurs l'un des rares programmes iconographiques complets du Moyen Âge sur la légende des Quinze Signes.

La traduction anglo-normande est relativement proche du modèle latin, et le traducteur parvient souvent à contourner les difficultés de sa source : pour le $3^{\mathrm{e}}$ signe par exemple, le verbe brere est utilisé afin de rendre la périphrase dabunt rugitus («ils pousseront un rugissement »); de même, dans le $5^{\mathrm{e}}$ signe, le verbe suer synthétise la périphrase dabunt rorem («ils donneront de la rosée »), tout en l'extrapolant. L'auteur précise également l'extension logique de l'hyperonyme aedificia (« édifices »): «mesuns et chasteus, musters, eglises e arbres». Cet exemple prouve que les médiévaux ne perdaient aucune occasion de s'abandonner à cette propension pour les énumérations. Mais ce souci de

${ }^{15}$ Holkham Bible Picture Book, éd. Frederick P. Pickering, Oxford, B. Blackwell, coll. « Anglo-Norman Text Society », 1971, p. 65-67. 
clarté se retourne parfois contre l'auteur: «les fendaces de la tere », par exemple, est une traduction pour le moins incongrue de cavernae.

Quoi qu'il en soit, le texte reste ici subordonné à l'image. L'artiste a eu le mérite de représenter visuellement des phénomènes qui a priori étaient plus faciles à saisir par l'imagination que par l'image : comment illustrer la lutte des pierres, la rosée ensanglantée ou la chute des étoiles ?

\section{Montpellier, Bibliothèque interuniversitaire, H 043, fol. 51vb- $52 a$}

La troisième version est la moins importante des cinq. Nous l'avons découverte au sein d'un manuscrit de la Bibliothèque interuniversitaire de Montpellier. Contemporain des deux versions précédentes, le manuscrit s'ouvre sur une traduction lorraine de la Consolation de Philosophie de Boèce, suivie d'une compilation de divers traités religieux, dont notre texte sur les Quinze Signes au fol. 51v. Il s'agit d'une traduction particulièrement concise, qui ne s'écarte à aucun moment de son modèle latin.

\section{«Selon ce que nostre Sire...»}

La quatrième version, qui a pour incipit «Selon ce que nostre Sire...», est plus intéressante ${ }^{16}$. Elle se trouve dans les manuscrits Paris, $\mathrm{BnF}$, français 1157, fol. 240rb-va (fin du XIII ${ }^{\mathrm{e}}$ siècle) et Bruxelles, Bibliothèque royale, 9106, fol. 253rb-vb ${ }^{17}$ (1475).

\footnotetext{
${ }^{16}$ Cette version a été éditée par Reine Mantou : voir «Le thème des "Quinze Signes $\mathrm{du}$ Jugement dernier" dans la tradition française », Revue belge de philologie et d'histoire, vol. 45, $\mathrm{n}^{\mathrm{o}} 3,1967$, p. 827-842, voir p. 840-841 (p. 840, il ne faut pas lire « fol. $25 \mathrm{r}^{\mathrm{O}} \mathrm{a}$ » mais « fol. $\left.25 \underline{3} \mathrm{r}^{\circ} \mathrm{a} »\right)$. Reine Mantou commet une erreur en déclarant que le manuscrit de Bruxelles, qu'elle édite, est « de la fin du XIII ${ }^{\mathrm{e}}$ siècle » (ibid., p. 831). La traduction remonte toutefois au XIII ${ }^{\mathrm{e}}$ siècle.

${ }^{17}$ Ce manuscrit a été copié dans la ville de Gand en 1475 par David Aubert pour la duchesse Marguerite d'York. Le colophon du manuscrit (fol. 256v) permet de connaître le contexte de production. On trouvera une transcription de ce colophon
} 
Le texte de Bruxelles contient une erreur pour le $1^{\mathrm{er}}$ signe («.xlv. coustees ${ }^{18} »$, alors qu'il faut lire «.xl.») et modifie légèrement le $7^{\mathrm{e}}$ signe, sûrement pour estomper l'invraisemblance de la «lithomachie»: «Le .vije . jour les roches et montaignes fonderont et cherront $^{19}$. ». De son côté, le texte de Paris commet une faute au $9^{\mathrm{e}}$ signe : «la $m e r$ sera yvee ${ }^{20} »$, lire « terre ».

Même si la traduction est globalement littérale, on observe quelques ajouts. Le $11^{\mathrm{e}}$ jour, l'apparition des ossements concernerait toutes les personnes qui sont mortes «depuiz le temps Adam $^{21} »$. Le $14^{\mathrm{e}}$ jour, le feu qui envahira le ciel et la terre aura pour fonction de purifier «les .iiij. elemenz ${ }^{22} »$. Enfin, le traducteur complète le $15^{\mathrm{e}}$ signe par un motif original, l'immobilisation des planètes ${ }^{23}$, que nous n'avons rencontré dans aucun autre texte : «et lez planetez qui ore sont en mouvement ne se mouvront; et sera le soleil en orient et la lune en occident $^{24}$.» Selon William Heist ${ }^{25}$, l'auteur s'appuierait sur le verset

dans Kurtis A. Barstow, «Appendix. The library of Margaret of York and some related books », dans Margaret of York, Simon Marmion and the Visions of Tondal, Malibu (Calif.), J. Paul Getty Museum, 1992, p. 257-263, voir p. 259. La majeure partie du manuscrit (fol.9r-199r) est consacrée à un texte majeur de la littérature didactique de la fin du XIII ${ }^{\mathrm{e}}$ siècle, La Somme le Roi. La fin du manuscrit compile une vingtaine de traités, le dernier étant réservé en toute logique au Jugement dernier.

${ }^{18}$ Bruxelles, Bibliothèque royale, 9106, fol. 253rb.

${ }^{19}$ Ibid. Cf. « Le .vij. lez pierrez s'entrebrisseront » : Paris, BnF, fr. 1157, fol. 240va.

${ }^{20}$ Ibid.

${ }^{21}$ Ibid. Le texte de Bruxelles ne donne pas cette précision, absente de la version de Pierre le Mangeur.

${ }^{22}$ Paris, BnF, fr. 1157, fol. 240va.

${ }^{23}$ Pour comprendre la cohérence de ce passage, il faut rappeler que l'astronomie médiévale considérait le soleil et la lune comme des planètes en mouvement. Dans son De sphaera mundi (c. 1230), Johannes de Sacrobosco intègre le soleil et la lune dans le groupe des planètes. Sur la question du soleil et de la lune au Moyen Âge, lire Le soleil, la lune et les étoiles au Moyen Âge, Aix-en-Provence, Université de Provence - CUERMA, coll. « Senefiance », 1983.

${ }^{24}$ Paris, BnF, fr. 1157, fol. 240va.

${ }^{25}$ William W. Heist, "The Fifteen Signs before the Judgement: further remarks », Mediaeval Studies, 22, 1960, p. 192-203, cit. p. 201. 
Lc 21, 25 : «et erunt signa in sole, et luna, et stellis ${ }^{26} »$. Reine Mantou conteste cette interprétation, sans proposer pour autant une autre explication ${ }^{27}$. Ce $15^{\mathrm{e}}$ signe dérive peut-être du bref épilogue sur le Jugement dernier qu'on pouvait déjà lire dans la version de Pierre le Mangeur : "Sicut fulgur exit ab oriente et paret usque in occidentem, sic erit adventus Filii hominis ${ }^{28} \gg$. La citation, extraite de l'Évangile selon Matthieu (Mt 24,27), concerne en réalité la parousie, l'adventus Filii hominis. La parousie sera aussi «fulgurante» qu'un éclair, fulgur, qui provient de l'orient et qui est vu jusqu'en occident. On peut supposer que le traducteur aura associé les deux points cardinaux que sont l'orient et l'occident au topos du soleil et de la lune, fréquent dans les descriptions du Jugement dernier. L’image qui en résulte n'en est que plus saisissante.

\section{Transmission de la légende dans le Dialogue du père et du fils}

La cinquième et dernière version, qui est de loin la plus complexe, se trouve dans un texte français inédit, intitulé le Dialogue du père et $d u$ fils. Bien que nous en ayons une trentaine de copies, ce texte n'a toujours pas été édité, et les travaux critiques qui en parlent sont relativement rares. Le Dialogue du père et $d u f^{\prime} i s^{29}$ est un manuel religieux à destination des laïcs. Probablement composé dans la première moitié du $\mathrm{XIII}^{\mathrm{e}}$ siècle, il fonctionne à la manière de l'Elucidarium d'Honorius Augustodunensis : un fils pose de nombreuses questions à son père, sur des sujets de religion et de morale comme le péché originel, la Trinité, l'Incarnation, les Sacrements, les miracles, l'Antichrist, les quinze signes

\footnotetext{
${ }^{26}$ «Et il y aura des signes dans le soleil, dans la lune et dans les étoiles. »

${ }^{27}$ Voir Reine Mantou, « Le thème des "Quinze signes du Jugement dernier"... », art. cit., p. 831.

28 «Comme l'éclair part de l'orient et apparaît jusqu'en occident, ainsi sera l'avènement du Fils de l'homme. »

${ }^{29}$ Ce titre apparaît dans plusieurs manuscrits : Paris, BnF, fr. 1136; fr. 13342 ; fr. 25408 , etc.
} 
du Jugement dernier, les péchés, la confession ou encore la charité. Paul Meyer fut l'un des premiers à aborder la transmission manuscrite de ce texte dans une notice sur un manuscrit de la bibliothèque municipale de Cambrai $^{30}$ (cote 256). Il y donne une liste de douze autres manuscrits contenant le Dialogue ${ }^{31}$. Mais il existe en réalité une trentaine de témoins pour ce texte. La plupart d'entre eux seront mentionnés ci-dessous ${ }^{32}$.

L'intérêt que présente le Dialogue du père et du fils est dû au nombre important de manuscrits que nous en avons, puisque de manière logique, plus la liste des Quinze Signes est copiée, plus elle se déforme. La légende des Quinze Signes n'ayant rien de canonique, qui plus est en langue vernaculaire, les copistes pouvaient se sentir tout à fait libres de réordonner cet inventaire de l'eschatologie populaire.

En regardant de près les manuscrits du Dialogue, on peut distinguer quatre groupes, selon le degré de réagencement qu'ils présentent par rapport au modèle latin. Nous les désignerons par les lettres $a, a^{\prime}, b$ et $c$. Seule la version a respecte l'ordre de Pierre le Mangeur. Les trois autres sont des versions fautives, probablement engendrées par des erreurs de copiste au cours de la transmission manuscrite. Notre lecteur trouvera à la fin de cet article un tableau qui synthétise l'ensemble des modifications.

La version $a$ figure dans les manuscrits suivants :

- Lyon, Bibliothèque universitaire, 15, fol. 85ra-b ;

- Paris, Arsenal, 2059, fol. 123r-v ;

- Paris, BnF, fr. 726, fol. 197va ; fr. 13342, fol. 20r-v ; fr. 25408, fol. $56 \mathrm{r}-\mathrm{v}$;

30 Paul Meyer, «Trois nouveaux manuscrits des sermons français de Maurice de Sully », Romania, 1899, 28, p. 245-268, voir p. 254-261 pour le Dialogue du père et du fils.

${ }^{31}$ Voir ibid., p. 258-259.

${ }^{32}$ Nous n'avons pas pu consulter le manuscrit Oxford, Bodleian Library, Fairfax 24, où le Dialogue se trouve aux fol.48v-61r, ni le manuscrit Reg. lat. 1668 de la Bibliothèque apostolique vaticane, qui donne une version abrégée du Dialogue aux fol. 64ra-73vb. 
- Soissons, Bibliothèque municipale, 224, fol. 15ra-va.

Cette version suit de près son modèle latin, à l'exception du premier jour: la mer, censée se dresser tel un mur (stans in loco suo quasi murus), se tiendra «comme s'el fust gelée ${ }^{33} »$. Peut-être le substantif murus a-t-il été confondu avec l'adjectif niveus. Cette confusion s'explique aisément d'un point de vue paléographique.

La version $a$ se caractérise également par sa conclusion, présente aussi dans la version $a^{\prime}$ : "Qui ce voudra crerre, si(l) le creie ; qui ne voudra, si [le] lest ${ }^{34}$. » Cette conclusion lapidaire confirme que la légende des Quinze Signes n'était pas toujours tenue pour parole d'évangile : bien qu'ils la recueillent, les médiévaux sont conscients de sa valeur d'apocryphe.

Dans l'un des six manuscrits du groupe $a$ (Paris, BnF, fr. 726), on observe une lacune. Le $4^{\mathrm{e}}$ signe (Incendie aquatique) n'a pas été recopié, soit parce que le copiste n'avait pas compris la phrase d'origine, soit parce que son modèle présentait lui-même une lacune. Cette lacune a donné naissance à un deuxième groupe $\left(a^{\prime}\right)$, qui réunit les quatre manuscrits suivants :

- Paris, Arsenal, 2071, fol. 52rb-va ;

- Paris, BnF, fr. 461, fol. 123rb-va ; fr. 1036, fol. 80rb-va ; fr. 12581 , fol. $355 \mathrm{vb}-356 \mathrm{ra}$.

L'omission du $4^{\mathrm{e}}$ signe a décalé d'un rang les signes suivants, mais ce décalage n'a concerné que les signes 5 à 10 , qui deviennent les signes 4 à 9, libérant ainsi la dixième place. Les quatre copistes tentèrent, chacun à leur manière, de résoudre cette lacune. Le copiste du manuscrit fr. 12581 est passé directement du signe 9 au signe 11, sans donner de signe 10. Le copiste du manuscrit fr. 1036 a choisi d'éluder cette lacune

\footnotetext{
${ }^{33}$ Paris, BnF, fr. 25408, fol. 56r.

${ }^{34}$ Ibid., fol. 56v. Corrigé d'après Paris, BnF, fr. 726, fol. 197va.
} 
en décalant les signes 10 à 15, libérant ainsi une place de choix, le dernier jour, lors duquel, selon lui, «sera tout consommé et parfet ${ }^{35}$.»Enfin, dans les manuscrits 2071 de l'Arsenal et fr. 461 de la BnF, le dixième signe a été créé de toutes pièces, à partir d'un motif répandu au Moyen Âge, celui de l'arbre renversé : «le $\mathrm{x}^{\mathrm{me}}$ jour s'esleveront toutes les racines des arbres vers le $\operatorname{ciel}^{36}{ } \gg$. Ce motif correspond en fait au septième jour du Dit des Quinze Signes ${ }^{37}$.

Les groupes $b$ et $c$ sont plus difficiles à appréhender. Le groupe $b$ est le plus représenté des quatre groupes, avec plus d'une dizaine de manuscrits :

- Bruxelles, BR, 10574-10585, fol. 91v-92r ;

- Cambrai, BM, 256, fol. 21va-22ra ;

- Lille, BM, 130, fol. 27rb-va ;

- Paris, BnF, fr. 1370, fol. 136v-137r ${ }^{38}$; fr. 1546, fol. 167ra-b ; fr. 1768 , fol. $89 \mathrm{v}-90 \mathrm{r}$; fr. 1850 , fol. $44 \mathrm{v}-45 \mathrm{r}$; fr. 25549 , fol. $52 \mathrm{r} 39$; n. a. f. 13521, fol. 276ra-b.

- Paris, Bibliothèque Sainte-Geneviève, 1654, fol. 133rb-va ;

- Salins-les-Bains, BM, 12, fol. 224v-225r.

Dans le groupe $b$, le décalage créé par la lacune du signe 4 est moins important que pour le groupe $a^{\prime}$, puisqu'il ne concerne que les signes 5 et 6 , devenus 4 et 5 . Nous pourrions penser que les groupes $a^{\prime}$ et $b$ sont deux branches indépendantes issues d'une lacune au sein du groupe $a$. Mais les choses ne sont pas si simples, car dans le groupe $b$, le

\footnotetext{
35 Paris, BnF, fr. 1036, fol. 80va.

${ }^{36}$ Paris, BnF, fr. 461, fol. 123va.

${ }^{37}$ Voir Les Quinze Signes du Jugement dernier, poème anonyme de la fin du XII ${ }^{e}$ ou $d u$ début du XIII siècle, éd. Erik von Kraemer, Helsinki, Societas scientiarum Fennica, 1966, v. 161-176.

${ }^{38}$ Le manuscrit fr. 1370 de la BnF ne donne que des extraits du Dialogue. Le texte sur les Quinze Signes s'écarte par endroits de la tradition: les signes 11 et 12 sont inversés, et le signe 14 est omis.

${ }^{39}$ Le début du texte manque dans le manuscrit fr. 25549.
} 
signe 6, qui désormais est vacant, reprend l'ancien signe 8 (Séisme), complété par le motif des arbres renversés (devenu 6 bis). Il existe donc une filiation entre le groupe $b$ et deux des manuscrits du groupe $a^{\prime}$ (les manuscrits 2071 de l'Arsenal et fr. 461 de la $\mathrm{BnF}$ ), dans lesquels nous trouvions déjà le motif de l'arbre renversé, même si ce n'était pas au jour 6 , mais au jour 10 . Toutefois, le groupe $b$ se distingue du groupe $a^{\prime}$ par l'ajout d'un autre motif, celui de l'obscurcissement du soleil et de la lune (12 bis) : « la lune et le soleil perdront leur clartée ${ }^{40}$.

Dans le groupe $c$, le désordre amorcé dans le groupe $b$ devient proprement chaotique. Ce groupe réunit les six manuscrits suivants :

- Avignon, BM, 344, fol. 35ra-va ;

- Chantilly, Musée Condé, 137, fol. 96vb-97va ; 138, fol. 64v-65r ;

- Paris, BnF, fr. 1136, fol. 68r-v ; n. a. f. 4338, fol. 91r-92v.

- Rouen, BM, 1052, fol. 78v-79r.

Dans ce groupe, le motif des arbres renversés disparaît, seul demeure l'ancien signe 8 (Séisme). Le signe 8 est lui-même remplacé par le signe 10 (Hommes fous). La place de ce dernier est occupée cependant par l'ancien signe 4 (Incendie aquatique), dont la disparition avait désordonné la liste d'origine dans la version $a^{\prime}$.

Que doit-on conclure de ce désordre qui progressivement, au fil de la transmission textuelle, s'immisce au sein de l'agencement conçu par Pierre le Mangeur ? Certes, on pourrait alléguer la notion de variance : un texte, au Moyen Âge, est destiné à une transformation perpétuelle. Mais ici, la variance n'est pas seulement due à des inadvertances dans le processus de copie. Il ne s'agit pas tant d'un désordre que d'une remise en ordre. Les copistes ont à leur disposition un inventaire, qu'ils jugent opportun de réordonner.

${ }^{40}$ Paris, BnF, fr. 1546 , fol. 167 rb. 
Cette idée est bien formulée, revendiquée même, par les copistes. Dans les manuscrits des groupes $a$ et $a^{\prime}$, le texte se termine par cette formule que nous avons déjà citée : «Qui ce voudra croire, si le croie, et qui ne voudra, si le lest ». La version $c$ s'achève sur un semblable sentiment d'incrédulité : «Et pour ce que il [les Quinze Signes] ne sont pas contenuz es livres des saintes escriptures qui sont aprouvées de sainte Esglise, qui ne les croira, ja pour ce ne se meffera ${ }^{41}$. »

Puisque le lecteur est libre, en l'absence de témoignage biblique, de ne pas croire à cette légende somme toute curieuse, il serait déplacé de jeter la pierre aux copistes pour avoir éprouvé la liberté d'en modifier indéfiniment l'ordre et la cohérence. Toutes proportions gardées, le désordre qui s'observe au fil de la transmission manuscrite reflète cette ambivalence dogmatique. Les médiévaux recopient inlassablement cet inventaire, mais ils se sentent autorisés à se l'approprier et à le réinventer dans la mesure où il n'a jamais fait autorité.

${ }^{41}$ Paris, BnF, fr. 1136, fol. 67r-v. 


\section{Tableau 3}

L'ordre des quinze signes dans les versions du Dialogue du père et du fils

\begin{tabular}{|l|l|l|l|l|}
\hline & $\boldsymbol{a}$ & $\boldsymbol{a}^{\prime}$ & $\boldsymbol{b}$ & $\boldsymbol{c}$ \\
\hline Crue & 1 & 1 & 1 & 1 \\
\hline Décrue & 2 & 2 & 2 & 2 \\
\hline Bêtes marines & 3 & 3 & 3 & 3 \\
\hline Incendie aquatique & 4 & & & 10 \\
\hline Rosée sanglante & 5 & 4 & 4 & 4 \\
\hline Chute des édifices & 6 & 5 & 5 & 5 \\
\hline Lutte des pierres & 7 & 6 & 7 & 7 \\
\hline Séisme & 8 & 7 & $6+8$ & 6 \\
\hline Nivellement & 9 & 8 & 9 & 9 \\
\hline Hommes fous & 10 & 9 & 10 & 8 \\
\hline Arbres renversés & & 10 & 6 bis & \\
\hline Ossements & 11 & 11 & 11 & 11 \\
\hline Chute des étoiles & 12 & 12 & 12 & 12 \\
\hline Soleil et lune obscurs & & & 12 bis & 12 bis \\
\hline Mort & 13 & 13 & 13 & 13 \\
\hline Conflagration & 15 & 14 & 14 & 14 \\
\hline Résurrection & & 15 & 15 \\
\hline & & & & \\
\hline
\end{tabular}

